\title{
The role of insulin as a key regulator of seeding, proliferation, and mRNA transcription of human pluripotent stem cells
}

\author{
Mohammad Shahbazi ${ }^{1}$, Paige Cundiff', Wenyu Zhou ${ }^{3,4,5}$, Philip Lee', Achchhe Patel², Sunita L. D'Souza², \\ Fahim Abbasi ${ }^{1}$, Thomas Quertermous ${ }^{1}$ and Joshua W. Knowles ${ }^{1,4^{*}}$ (D)
}

\begin{abstract}
Background: Human-induced pluripotent stem cells (hiPSCs) show a great promise as a renewable source of cells with broad biomedical applications. Since insulin has been used in the maintenance of hiPSCs, in this study we explored the role of insulin in culture of these cells.

Methods: We report conditions for insulin starvation and stimulation of hiPSCs. Crystal violet staining was used to study the adhesion and proliferation of hiPSCs. Apoptosis and cell cycle assays were performed through flow cytometry. Protein arrays were used to confirm phosphorylation targets, and mRNA sequencing was used to evaluate the effect of transcriptome.

Results: Insulin improved the seeding and proliferation of hiPSCs. We also observed an altered cell cycle profile and increase in apoptosis in hiPSCs in the absence of insulin. Furthermore, we confirmed phosphorylation of key components of insulin signaling pathway in the presence of insulin and demonstrated the significant effect of insulin on regulation of the mRNA transcriptome of hiPSCs.

Conclusion: Insulin is a major regulator of seeding, proliferation, phosphorylation and mRNA transcriptome in hiPSCs. Collectively, our work furthers our understanding of human pluripotency and paves the way for future studies that use hiPSCs for modeling genetic ailments affecting insulin signaling pathways.
\end{abstract}

Keywords: Insulin, Insulin signaling, Proliferation, Apoptosis, Pluripotent stem cells, Transcriptional regulation

\section{Background}

Human-induced pluripotent stem cells (hiPSCs) can be expanded continuously and can differentiate into all lineages of the human body. Due to these characteristics, hiPSCs provide a renewable source of cells for developmental studies, drug screening, and regenerative medicine [1]. Additionally, undifferentiated hiPSCs can recapitulate the aspects of genetic diseases provided that the affected cellular and molecular pathways are active in the

\footnotetext{
* Correspondence: knowlej@stanford.edu

'Stanford Cardiovascular Medicine and Cardiovascular Institute, Stanford School of Medicine, Stanford University, Falk CVRC, Room CV273, MC 5406 300 Pasteur Drive, Stanford, CA 94305, USA

${ }^{4}$ Stanford Diabetes Research Center, Stanford University, Stanford, CA 94305, USA

Full list of author information is available at the end of the article
}

pluripotent cells [2-4]. Since the initial description of human-induced pluripotent stem cells $[5,6]$, there has been a growing interest in identification of essential components of their culture. Studying these key components and the respective signaling pathways improves our understanding of pluripotency and also demonstrates the potentials and limitations of hiPSCs in modeling of diseases associated with cell signaling.

Transforming growth factor beta 1 (TGFB1) and fibroblast growth factor 2 (FGF2) are two of the components that have been identified that seem to be essential in the maintenance of human pluripotent stem cells. Another essential component is insulin, a peptide hormone that regulates an intricate network of signaling pathways and cellular responses [7] and has been identified as one of

(c) The Author(s). 2019 Open Access This article is distributed under the terms of the Creative Commons Attribution 4.0 International License (http://creativecommons.org/licenses/by/4.0/), which permits unrestricted use, distribution, and 
the key ingredients in culture of human pluripotent stem cells [8-10]. However, the role of insulin in the culture of hiPSCs is not fully understood. In this study, we report conditions for insulin starvation of hiPSCs for up to 3 days and use this setting to explore the effect of insulin on several cellular and molecular functions that have been associated with insulin signaling as well as explore the effect of insulin on global mRNA transcription in hiPSCs.

\section{Methods}

\section{Culture of hiPSCs}

hiPSC lines were derived from PBMCs using integrationfree Sendai virus to deliver the Yamanaka factors as reported earlier [11]. These cell lines were initially expanded on matrigel in the presence of mTeSR1 (StemCell Technologies) and passaged using $0.5 \mathrm{mM}$ EDTA. All cells were then thawed and adapted to TeSR-E8 for at least 2 weeks prior to the experiments. The medium was changed daily starting within the initial 3 days of culture. To prepare an insulin-free hPSC medium, mTeSR-E5-defined medium (StemCell Technologies) was supplemented with FGF2 (bFGF, PeproTec, final concentration of $100 \mu \mathrm{g} / \mathrm{l}$ ) and TGFB1 (PeproTec, final concentration of $2 \mu \mathrm{g} / \mathrm{l}$ ). Cells were washed 4 times with DMEM-F12 (Life Technologies) prior to insulin starvation experiments. When insulin treated groups were needed for the experiments, the medium was further supplemented with insulin (insulin, human recombinant, zinc solution; Life Technologies) to the final concentration of $19.4 \mathrm{mg} / \mathrm{l}$. During maintenance in TeSR-E8 and experiments, the cultures were fed double medium from day 4 of culture onwards, unless the cells were used for overnight experiments on day 4 .

Of the four hiPSC lines initially used in the preliminary experiments (Additional file 4: Table S1), one line showed sporadic patches of differentiating colonies (data not shown). While the vast majority of the cells maintained their pluripotent morphology in the line, we excluded this line from our experiments to prevent the chance that traces of differentiated cells interfere with our data analysis.

\section{Immunofluorescence staining}

Primary and secondary antibodies used for pluripotency were obtained from a Pluripotent Stem Cell 4-Marker Immunocytochemistry Kit (Life Technologies). DAPI was used for counter staining of the nuclei. Secondary antibody stainings were used as controls (data not shown).

\section{Crystal violet staining}

Crystal violet staining was used for visualization of the cells in quantification of cell adhesion and cell expansion. Samples were fixed with $4 \%$ PFA in PBS for $5 \mathrm{~min}$ and were stained with $0.05 \%$ crystal violet solution for $30 \mathrm{~min}$; plates were rinsed up to 4 times with water, dried, and were used for imaging. The staining in the wells was quantified using microscopic imaging of the wells for seeding experiments, or direct images of the wells for expansion experiments. The percentage of surface area covered by cells (stained regions) was measured using photoshop and was compared between groups.

\section{Cell cycle assay}

Cells were harvested with accutase (Millipore) or Stempro Accutase (Life Technologies), fixed with $70 \%$ ethanol and stained through incubation of cells in PBS- $0.1 \%$ Triton-X solution supplemented with $0.2 \mathrm{mg} / \mathrm{ml}$ RNase $\mathrm{A}$ and $0.02 \mathrm{mg} / \mathrm{ml}$ propidium iodide (PI) at room temperature for $30 \mathrm{~min}$. Prepared samples were analyzed through flow cytometry. FlowJo software (Treestar, Inc) was used for modeling and analysis of the cell cycle.

\section{Apoptosis assay}

Cells were harvested with accutase. Supernatant and rinse solutions were collected separately and were pooled with the harvested cells prior to staining. Samples were stained using dead cell apoptosis kit with Annexin V FITC and PI (Life Technologies) and analyzed through flow cytometry.

\section{Protein arrays}

Human Phospho-RTK Antibody Array, Human PhosphoMAPK Antibody Array, Human Phospho-Kinase Antibody Array, and Human Apoptosis Antibody Array Kits were obtained from $R \& D$ system and used according to the provided instructions. Briefly, lysates were harvested after treatment of cells in the described experimental conditions. The cells were rinsed once with PBS (with $\mathrm{Ca} / \mathrm{Mg}$ ) and lysed with inhibitors supplemented with lysis buffer. Concentration of protein lysates were subsequently determined by $\mathrm{BCA}$ assay. The membranes were incubated overnight with protein lysate at $4{ }^{\circ} \mathrm{C}$ on a rocking platform before incubation with a secondary antibody or directly with a detection reagent based on the array manual provided with the kit.

Li-Cor Odyssey imager and Image Studio software (Li-Cor Biosciences) were used for imaging and quantification of protein array results. Image Studio software was used to quantify the signal of the array dots. The data were normalized in each dataset through division of signal value obtained from groups treated without insulin to their respective insulintreated groups.

\section{RNA extraction and RNA sequencing}

RNA extraction and RNA sequencing were performed as described earlier (Carcamo-Orive et al., 2017). Sequencing reads were first quality checked by FastQC (v 0.11.8). 
Reads above Q30 were further mapped and reads per gene were counted by STAR (v2.7). Read count normalization and differential analyses were performed using Deseq2 $\mathrm{R}$ package (v1.22.2) with custom codes. Pathway enrichments were performed using Gene Set Enrichment Analysis (GSEA) with MSigDB 6.2 database. For $24 \mathrm{~h}$, all significantly $\left(p \_\right.$adj $<0.05$ ) differential genes were included for the enrichment analysis, while for $72 \mathrm{~h}$, the significantly ( $p \_$adj $<5$ E10-4) differential genes were included to obtain a similar number of significant genes as compared to those under $24 \mathrm{~h}$ for the enrichment analysis.

\section{Results}

\section{The effect of insulin on seeding of hiPSCs}

To investigate the effect of insulin on seeding of hiPSC aggregates, colonies were harvested by EDTA and were seeded with or without insulin. The attachment of the hiPSC aggregates was evaluated microscopically after crystal violet staining of plates 1 day after seeding. The presence of insulin significantly improved the seeding of hiPSC aggregates (Fig. 1a, b).

To determine the effect of insulin on seeding of dissociated hiPSCs, the colonies were digested with Accutase and passed through the $40-\mu \mathrm{M}$ cell strainer and were used for crystal violet staining. The presence of Y-27632, which is an inhibitor of rho-associated kinase (ROCK), prevents apoptosis in dissociated hiPSCs [12]. Our results demonstrate that the presence of both Y-27632 and insulin are essential for attachment of dissociated hiPSCs (Fig. 1c, d). Inclusion of either component allowed very limited attachment of cells while no attached cells were observed when both reagents were absent.

The effect of insulin on pluripotent morphology, expression of pluripotency markers, and expansion of hiPSC colonies

To compare the effect of insulin on pluripotent morphology and expression of pluripotency markers, 3 lines of hiPSCs
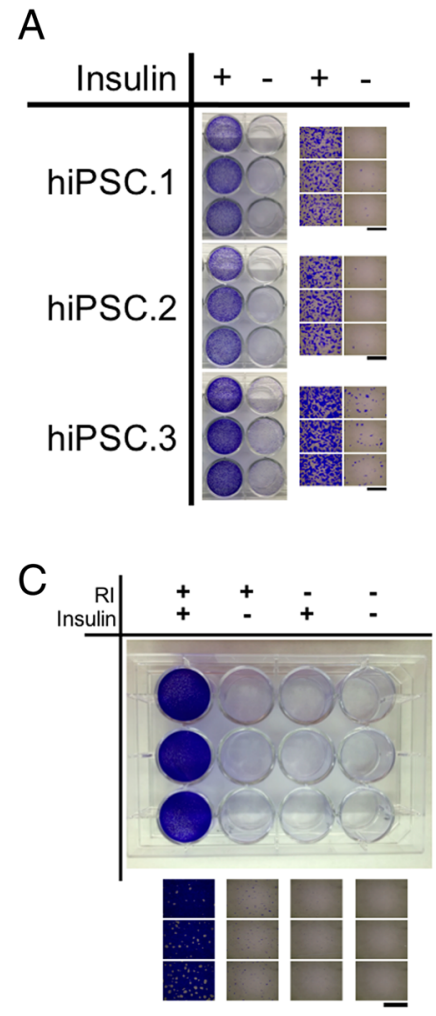

\section{B}

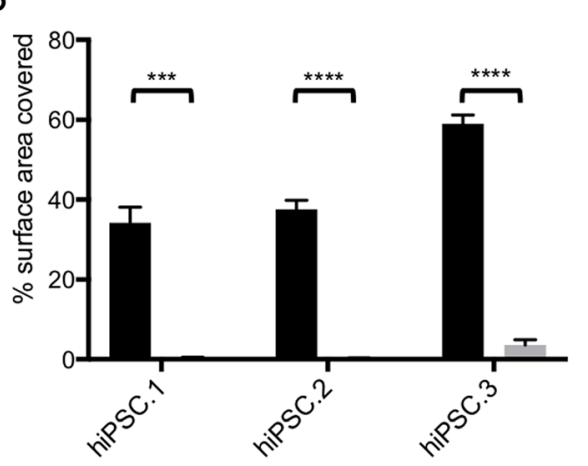

D

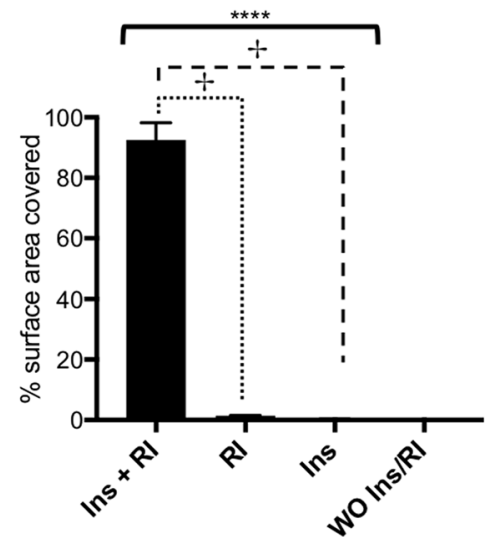

Fig. 1 The effect of insulin on seeding of hiPSCs. hiPSCs were harvested with EDTA as cell aggregates and were imaged 1 day after seeding. a Whole-well images and microscopic images from the center of each well after crystal violet staining. b Quantification of the percentage surface area covered by hiPSC fragments for the microscopic images from $\mathbf{a}$. Data represented as mean \pm SD. Two-tailed Student's $t$ test was used for comparison of groups, each cell line in triplicates. hiPSC.3 colonies were harvested with accutase as single cells/small cell fragments and were passed through a 40- $\mu$ m filter. c Whole-well images and microscopic images of the center of each well after crystal violet staining. $\mathbf{d}$ Quantification of percentage of surface area covered by hiPSCs for the microscopic images from $\mathbf{c}$. Data represented as mean \pm SD. One-way ANOVA test was used between first three groups; no adherent cells were detected in the last group. ${ }^{* *} p<0.001 .{ }^{* * *} p<0001$. +Significant difference between groups in LSD test. Experiment in triplicates. Scale bar: 2 mm. Rl: rock inhibitor 
(Additional file 4: Table S1) were maintained in culture for 3-4 days and were subsequently cultured with or without insulin for 3 additional days. These cells maintained pluripotent morphology (Fig. 2a) and expression of key pluripotency markers SOX2, POU5F1, and TRA-1-60 after 3 days of insulin starvation (Fig. 2b).

The surface area of the tissue culture plate covered by hiPSC colonies in the presence of insulin was significantly larger than the surface area covered in the absence of insulin (Fig. 2c, d). These observations were in line with number of cells in each condition, determined by manual cell count (Fig. 2e) and indicate that the presence of insulin significantly improves the proliferation of hiPSCs.
Insulin changes the cell cycle profile and inhibits apoptosis in hiPSCs

To investigate the effect of insulin on cell cycle, the cells were cultured with or without insulin for 3 days and then used for flow cytometry-based cell cycle assay using propidium iodide (PI) staining. There was a significant decrease in the percentage of the cells at the G1 phase is the absence of insulin (Fig. 3a, b).

After hiPSCs were cultured overnight (16-18 h) with and without insulin, there was a significant increase in detected apoptotic cells in the absence of insulin in a flow cytometry-based apoptosis assay (Fig. 3c, d).

Protein array analysis of apoptotic proteins after $16 \mathrm{~h}$ of insulin starvation demonstrates more than a 3-fold

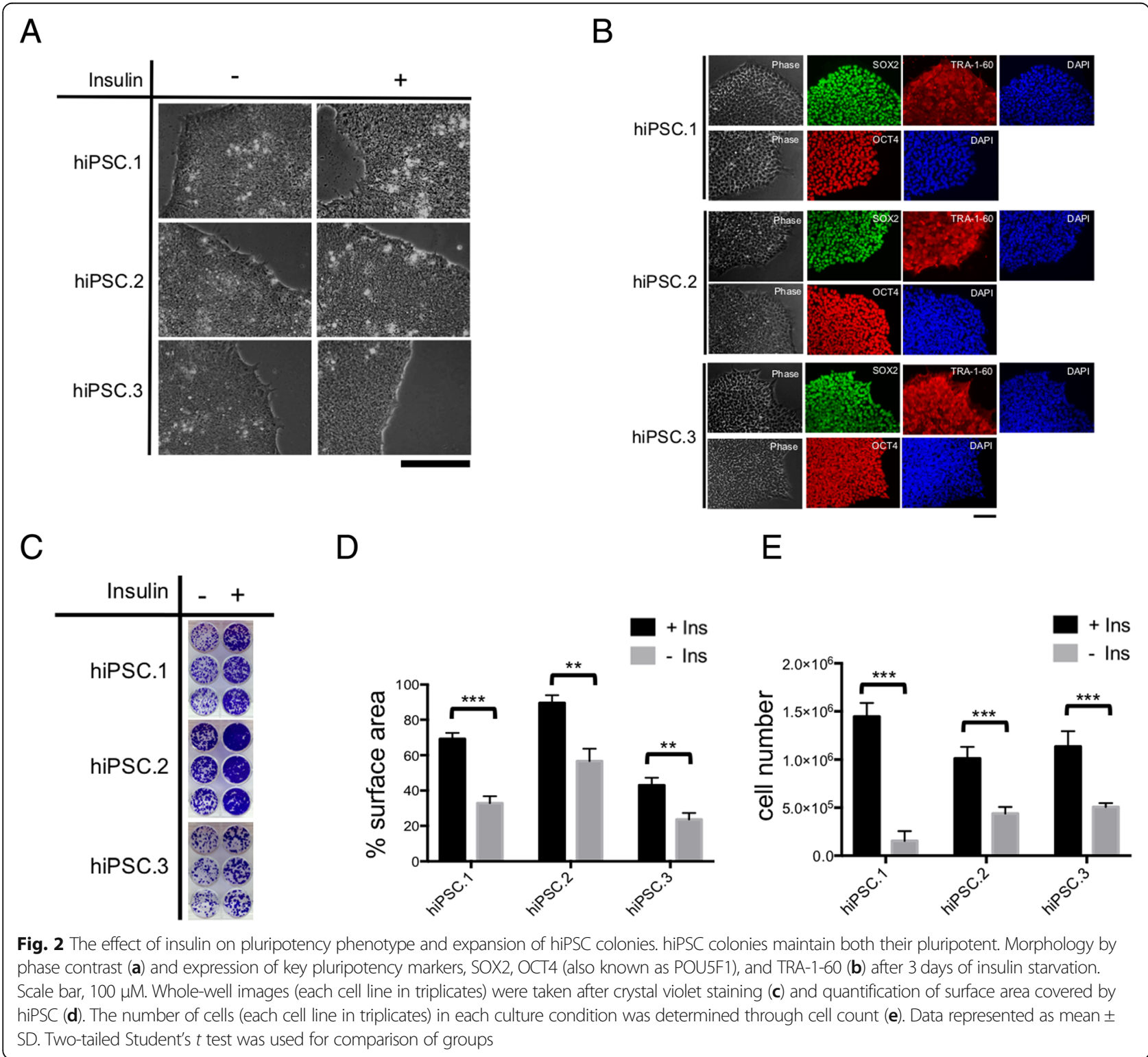




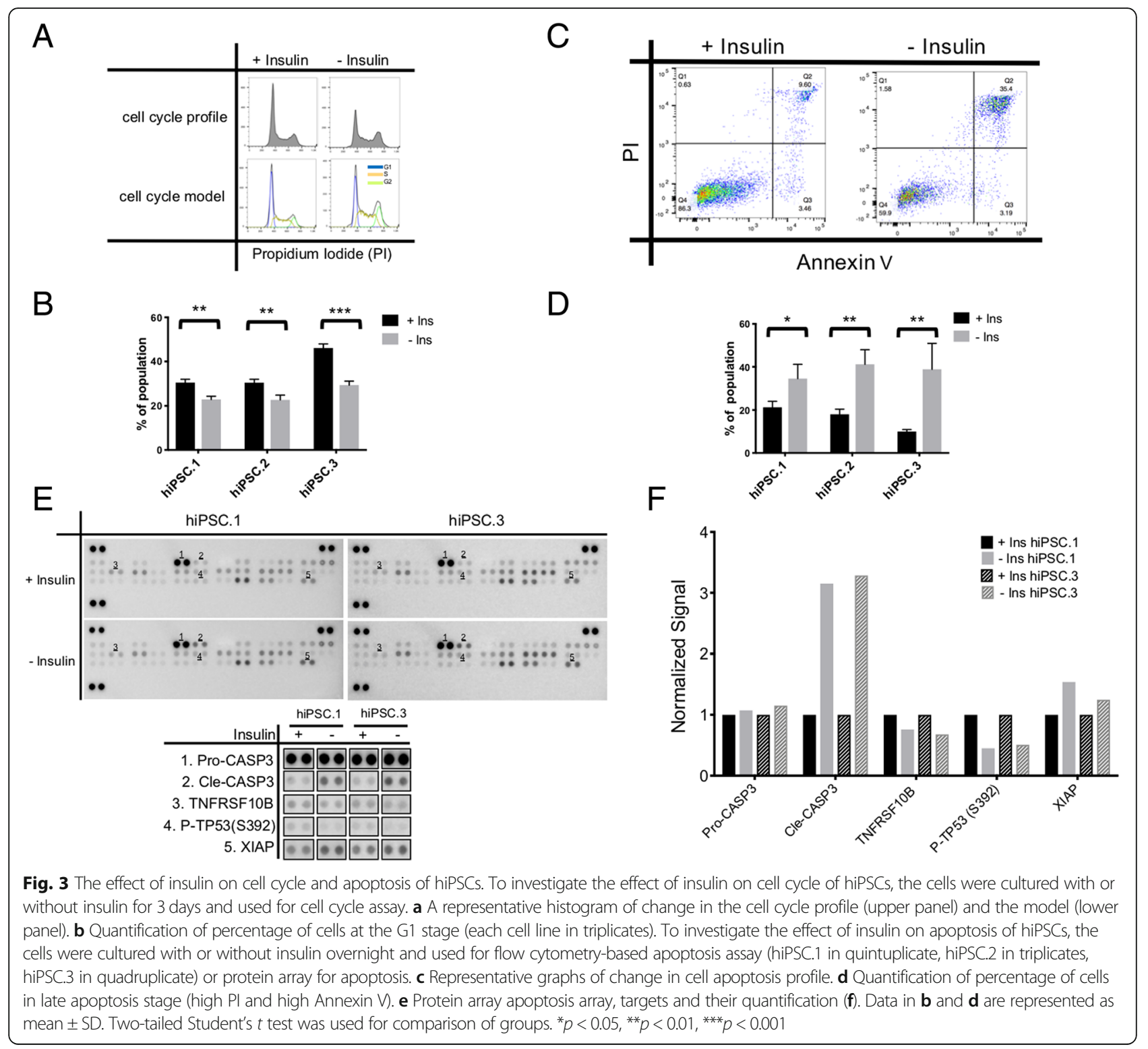

increase in levels of cleaved caspase- 3 while levels of pro-caspase-3 remain at similar levels (Fig. 3e, f). This robust increase in levels of cleaved caspase- 3 is accompanied by reduction in XIAP, TNFRSF10B proteins, and serine 392 phosphorylated TP53.

\section{Verification of insulin-mediated protein phosphorylation in hiPSCs}

We used protein arrays to monitor phosphorylation of a set of tyrosine kinase receptors and phosphokinases (Fig. 4). We confirmed that the insulin receptor (INSR) and insulin-like growth factor 1 receptor (IGF1R) are highly phosphorylated in the presence of insulin. Furthermore, our results indicate that phosphorylation of AKT and WNK1 and GSK3 are regulated by insulin stimulation in hiPSCs.
Insulin regulates transcriptome of pluripotent stem cells To gain an understanding of the transcriptional changes induced by insulin in hiPSCs, we looked at the effect of insulin on gene expression at $24 \mathrm{~h}$ and $72 \mathrm{~h}$.

An average of 62.7 million reads were obtained for each sample, and the average genome mapping rate was 90\% (Additional file 1: Figure S1 and Additional file 2: Figure S2). The number of identified genes is shown in Additional file 4: Table S2.

The prominent effect of insulin on global mRNA transcription of the cells is demonstrated by the t-SNE plot (Fig. 5a) and ternary plot (Fig. 5b). The t-SNE plot clearly indicates that the presence of insulin has a stronger global effect on transcription than internal variations across the cells at $72 \mathrm{~h}$. This is manifested by closer clustering of samples based on insulin treatment rather than 


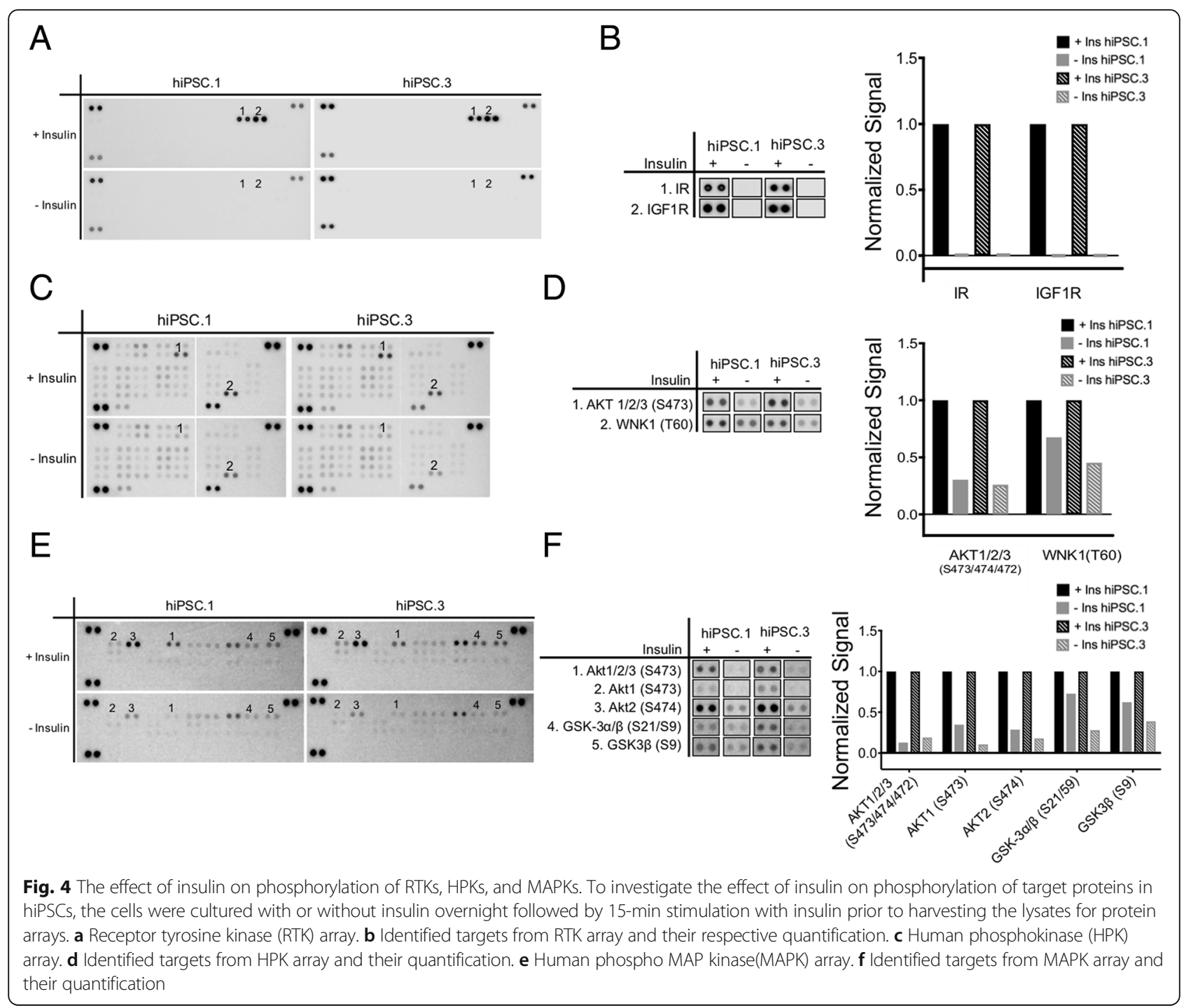

the individual lines at $72 \mathrm{~h}$ time point. A ternary plot shows that among the three major variables (time, cell line, or treatment), insulin treatment explains most of the variances at a majority of the mapped genes.

We identified 1161 differentially expressed genes at 24 $\mathrm{h}$ of hiPSC culture with and without insulin and 6753 differentially expressed genes at $72 \mathrm{~h}$, with $q$ value < 0.05 . The 20 top differentially expressed genes at $24 \mathrm{~h}$ and $72 \mathrm{~h}$ time points are listed in Additional file 4: Tables S3 and S4. The relationship between $\log 2$ fold change and $p$ values is shown as volcano plots for each time point with differentially expressed genes shown in red (Fig. 5c). The heatmaps of differentially expressed genes at $24 \mathrm{~h}$ and $72 \mathrm{~h}$ are shown in Fig. $5 \mathrm{~d}$.

We compared the effect of insulin on gene expression at $24 \mathrm{~h}$ and $72 \mathrm{~h}$. There is a strong correlation between gene expression at $24 \mathrm{~h}$ and $72 \mathrm{~h}$ suggesting that the effect of insulin on transcriptome is generally consistent between $24 \mathrm{~h}$ and 72 with more prominent effect at $72 \mathrm{~h}$ (Fig. 6a). This consistency is also demonstrated by overlap of genes differentially expressed at $24 \mathrm{~h}$ with $72 \mathrm{~h}$ (Fig. 6b).

To better understand the affected biological processes using identified differentially expressed genes, we performed Gene Ontology (GO) term enrichment for biological processes at each time point.

The top 25 terms that were associated with differentially expressed transcripts at $24 \mathrm{~h}$ are shown in Table 1 . The top 25 biological Gene Ontology (GO) processes terms that were associated with significantly differentially expressed transcripts at $72 \mathrm{~h}$ are shown in Additional file 4: Table S5. From the list of top 20 GO processes at $24 \mathrm{~h}$, we selected the high-ranked terms associated with our results and heatmaps of differentially expressed genes within each term were generated (Additional file 3: Figure S3). 


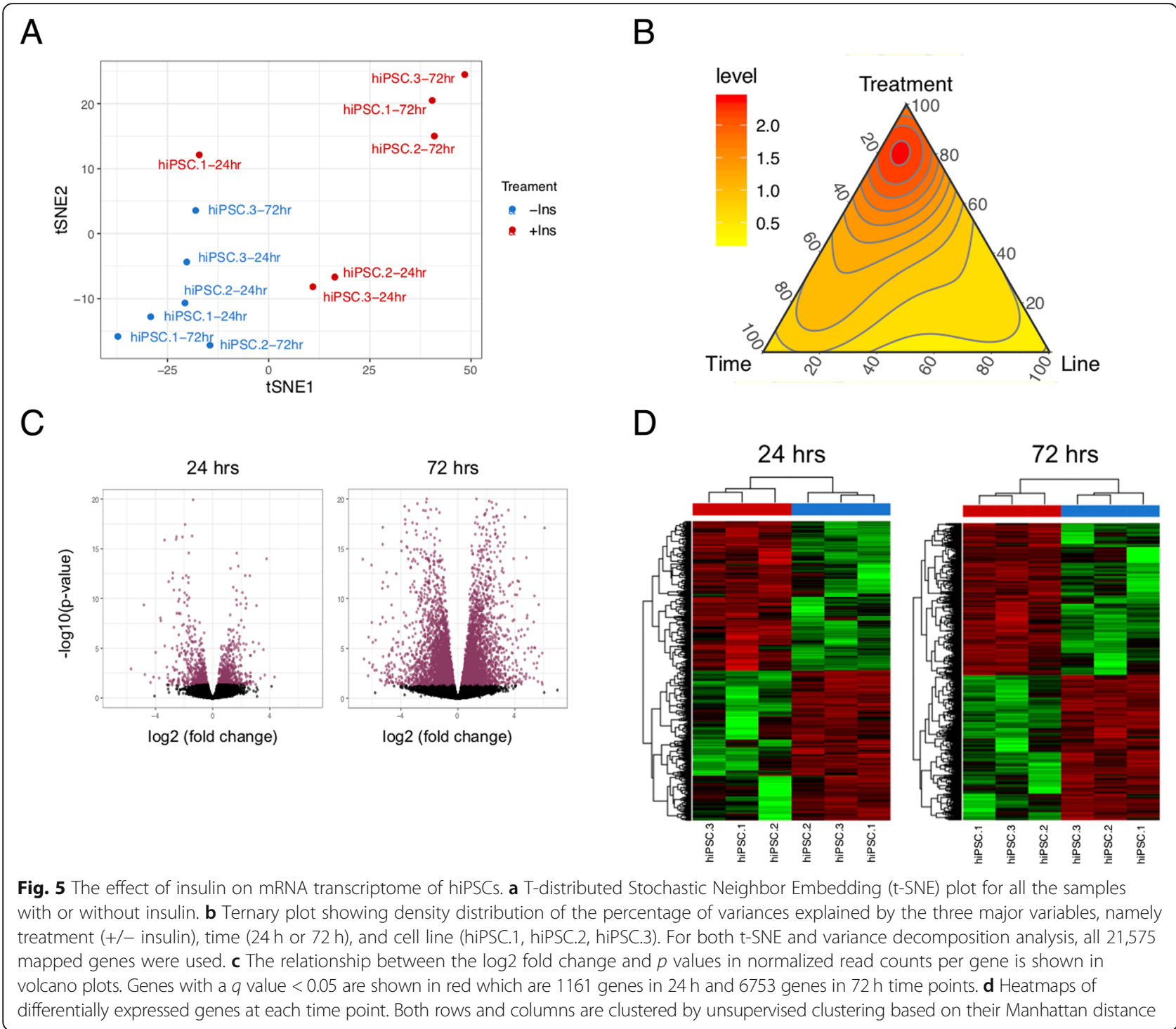

We compared differentially expressed genes at $24 \mathrm{~h}$ and $72 \mathrm{~h}$ with KEGG pathway database to investigate the effect of insulin on expression of known genes involved in pathways regulating the pluripotency (Additional file 4: Table S6) and insulin signaling pathway (Additional file 4: Table S7).

\section{Conclusion}

We investigated the effect of insulin on seeding of hiPSC aggregates or dissociated hiPSCs. Our results with dissociated hiPSCs demonstrate that insulin exerts its effect on their seeding independent of Y-27632. This observation is in line with an earlier report indicating that insulin is essential for survival of dissociated human pluripotent stem cells [8]. We expand on this by also demonstrating the significant effect of insulin on seeding of hiPSC aggregates without use of either chemical inhibitors blebbistatin or Y-27632.

Our data indicate that insulin significantly improves the expansion of hiPSC colonies and cellular proliferation. These results are broadly in line with earlier reports [8], although we observe less drastic effect after insulin starvation in our experiments on the expansion of the cells, possibly because Chen et al. used dissociated cells rather than hiPSC aggregates in their experiments. In our work, we also investigated the effect of insulin in expansion of hiPSC colonies without using of blebbistatin or Y-27632 and separated the seeding experiments from expansion experiments.

Specific inhibition of IGF1R with shRNA in human embryonic stem cells (hESCs) has been reported to lead to their competitive disadvantage in proliferation [10], demonstrating the importance of IGF1R in expansion of 
A

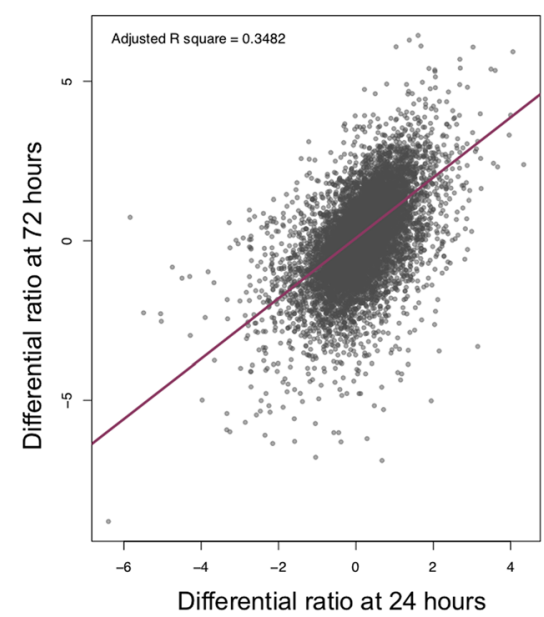

B

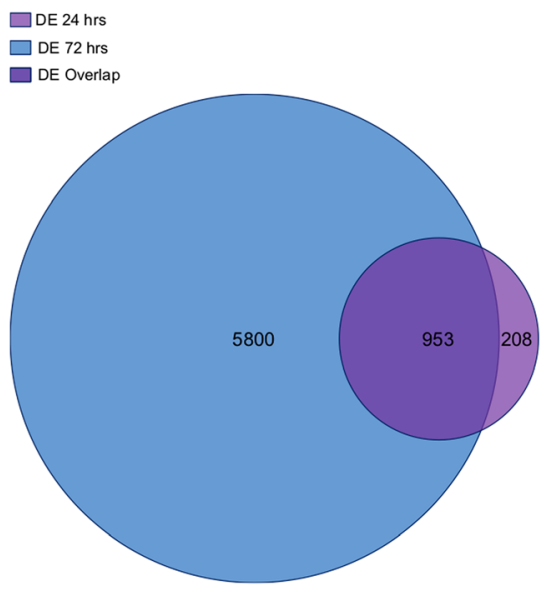

Fig. 6 Comparison of gene expression at 24 and $72 \mathrm{~h}$ of hiPSC culture with or without insulin. a Scatter plot showing the correlation between the log 2 fold change (+ insulin/- insulin) at $24 \mathrm{~h}$ versus $72 \mathrm{~h}$, with a $p$ value $<2.2 \mathrm{e}-16$. All genes were included for this comparison. $\mathbf{b}$ The number of overlapping and unique genes that were differentially expressed between the two time points by Venn diagram

human pluripotent stem cells. In addition, hiPSCs derived from insulin-resistant patients with mutations in their insulin receptor have demonstrated lower proliferation compared to their normal counterparts in the presence of insulin [2], suggesting that insulin signaling plays a regulatory role in proliferation of hiPSCs. Phosphorylation of IGF1R and INSR in the presence of conditioned medium containing high levels of insulin [10] or after insulin stimulation [2] has also been reported. Consistent with these studies, we observed robust phosphorylation of both the INSR as well as IGF1R. In addition, our data appear to demonstrate a more robust phosphorylation of these receptors, potentially due to different starvation conditions or higher concentrations of insulin used.

Cell division and cell death are two main contributory factors to cellular proliferation. We observed a significant increase in apoptosis by hiPSCs in the absence of insulin. This observation was consistent with the detection of higher levels of cleaved caspase-3. Caspase-3 plays a key role in execution stage of apoptosis. Interestingly, cleavage of pro-caspase-3 has been reported after inhibition of AKT phosphorylation in hiPSCs [13]. In addition, we detected lower levels of TNFRSF10B after insulin starvation. TNFRSF10B has been reported to play a dual role in cell survival [14].

After insulin starvation, we detected lower levels of serine 392 phosphorylated TP53 and higher level of XIAP in an apparent contrast to the usual role attributed to them. Serine 392 phosphorylated TP53 has been reported to contribute to genotoxic stress and apoptosis [15]. XIAP has also been identified as an anti-apoptotic molecule; nevertheless, upregulation of XIAP in response to environmental stress stimuli to induce apoptosis resistance has been reported in a cellular model [16]. These observations can be potentially attributed to the coping mechanism in surviving cells in response to the activated apoptotic pathways.

Phosphorylation of AKT is a critical node in insulin signaling [7]. AKTs play a central role in pluripotency and it has been demonstrated that overexpression of AKT improves the maintenance of pluripotent morphology in a primate pluripotent stem cell model [17]. Our data confirms that AKTs including AKT1 and AKT2 are main targets of insulin signaling in hiPSCs, consistent with earlier reports [18]. We also demonstrate that phosphorylation of GSK3B is a target of insulin stimulation in hiPSCs. Inhibition of phosphorylation of both AKT and GSK3B has been demonstrated to increase apoptosis in hiPSCs $[13,19]$.

Despite phosphorylation of AKT and GSK3B in the presence of insulin, we still detected a basal level of phosphorylation of these target proteins in the absence of insulin. This observation is in line with earlier reports of contribution of other growth factors including endogenously secreted ELABELA [18] and high concentrations of FGF [20] in the medium which contribute independently to activation of AKT signaling pathway in hiPSCs.

With regard to cell cycle profile of hiPSC populations after 3 days of insulin starvation, we observed a significant reduction in percentage of cells in the G1 phase. This change in cell cycle profile is similar to reports regarding inhibition of phosphorylation of either GSK3B (which we here demonstrate to be a target of insulin signaling in hiPSCs) or receptor tyrosine kinase ERBB2 in human pluripotent stem cells. In both studies, inhibition 
Table 1 The top 25 significant GO (biological process) terms associated differentially expressed transcripts at $24 \mathrm{~h}$ of hiPSC culture with or without insulin

\begin{tabular}{|c|c|c|c|c|c|c|}
\hline & GO term & $\begin{array}{l}\text { \# Genes in gene set } \\
(\mathrm{K})\end{array}$ & $\begin{array}{l}\text { \# Genes in overlap } \\
\text { (k) }\end{array}$ & $\mathrm{k} / \mathrm{K}$ & $p$ value & $\begin{array}{l}\text { FDR } q \\
\text { value }\end{array}$ \\
\hline 1 & GO_REGULATION_OF_INTRACELLULAR_SIGNAL_TRANSDUCTION & 1656 & 148 & 0.0894 & $7.93 \mathrm{E}-48$ & $3.52 \mathrm{E}-44$ \\
\hline 2 & GO_TISSUE_DEVELOPMENT & 1518 & 130 & 0.0856 & $6.17 \mathrm{E}-40$ & $1.37 \mathrm{E}-36$ \\
\hline 3 & GO_CELL_DEVELOPMENT & 1426 & 125 & 0.0877 & $2.05 \mathrm{E}-39$ & $3.03 \mathrm{E}-36$ \\
\hline 4 & GO_REGULATION_OF_PHOSPHORUS_METABOLIC_PROCESS & 1618 & 133 & 0.0822 & $5.80 \mathrm{E}-39$ & $6.43 \mathrm{E}-36$ \\
\hline 5 & GO_POSITIVE_REGULATION_OF_RESPONSE_TO_STIMULUS & 1929 & 139 & 0.0721 & $1.52 \mathrm{E}-34$ & $1.35 \mathrm{E}-31$ \\
\hline 6 & GO_RESPONSE_TO_EXTERNAL_STIMULUS & 1821 & 134 & 0.0736 & $3.23 \mathrm{E}-34$ & $2.39 \mathrm{E}-31$ \\
\hline 7 & GO_REGULATION_OF_CELL_PROLIFERATION & 1496 & 120 & 0.0802 & $4.09 \mathrm{E}-34$ & $2.59 \mathrm{E}-31$ \\
\hline 8 & $\begin{array}{l}\text { GO_POSITIVE_REGULATION_OF_MULTICELLULAR_ORGANISMAL_ } \\
\text { PROCESS }\end{array}$ & 1395 & 115 & 0.0824 & $8.90 \mathrm{E}-34$ & 4.93E-31 \\
\hline 9 & GO_NEUROGENESIS & 1402 & 115 & 0.082 & $1.41 \mathrm{E}-33$ & $6.93 \mathrm{E}-31$ \\
\hline 10 & GO_INTRACELLULAR_SIGNAL_TRANSDUCTION & 1572 & 122 & 0.0776 & $2.60 \mathrm{E}-33$ & $1.15 \mathrm{E}-30$ \\
\hline 11 & GO_POSITIVE_REGULATION_OF_CELL_COMMUNICATION & 1532 & 120 & 0.0783 & $3.85 E-33$ & $1.55 \mathrm{E}-30$ \\
\hline 12 & GO_REGULATION_OF_CELL_DEATH & 1472 & 117 & 0.0795 & $6.77 \mathrm{E}-33$ & $2.50 \mathrm{E}-30$ \\
\hline 13 & $\begin{array}{l}\text { GO_REGULATION_OF_MULTICELLULAR_ORGANISMAL_ } \\
\text { DEVELOPMENT }\end{array}$ & 1672 & 125 & 0.0748 & $1.46 \mathrm{E}-32$ & $4.98 \mathrm{E}-30$ \\
\hline 14 & GO_RESPONSE_TO_ENDOGENOUS_STIMULUS & 1450 & 115 & 0.0793 & $3.00 \mathrm{E}-32$ & $9.51 \mathrm{E}-30$ \\
\hline 15 & GO_CIRCULATORY_SYSTEM_DEVELOPMENT & 788 & 83 & 0.1053 & $7.93 \mathrm{E}-32$ & $2.35 \mathrm{E}-29$ \\
\hline 16 & GO_PHOSPHATE_CONTAINING_COMPOUND_METABOLIC_PROCESS & 1977 & 135 & 0.0683 & $3.57 \mathrm{E}-31$ & $9.89 \mathrm{E}-29$ \\
\hline 17 & GO_REGULATION_OF_PROTEIN_MODIFICATION_PROCESS & 1710 & 124 & 0.0725 & $4.79 E-31$ & $1.25 \mathrm{E}-28$ \\
\hline 18 & GO_REGULATION_OF_TRANSPORT & 1804 & 127 & 0.0704 & $1.44 \mathrm{E}-30$ & $3.55 \mathrm{E}-28$ \\
\hline 19 & GO_MOVEMENT_OF_CELL_OR_SUBCELLULAR_COMPONENT & 1275 & 104 & 0.0816 & $3.45 \mathrm{E}-30$ & 8.05E-28 \\
\hline 20 & GO_POSITIVE_REGULATION_OF_GENE_EXPRESSION & 1733 & 123 & 0.071 & $6.25 \mathrm{E}-30$ & $1.39 \mathrm{E}-27$ \\
\hline 21 & GO_CELLULAR_RESPONSE_TO_ORGANIC_SUBSTANCE & 1848 & 127 & 0.0687 & $1.41 \mathrm{E}-29$ & $2.98 \mathrm{E}-27$ \\
\hline 22 & GO_POSITIVE_REGULATION_OF_MOLECULAR_FUNCTION & 1791 & 124 & 0.0692 & $3.56 \mathrm{E}-29$ & $7.18 \mathrm{E}-27$ \\
\hline 23 & GO_ENZYME_LINKED_RECEPTOR_PROTEIN_SIGNALING_PATHWAY & 689 & 73 & 0.106 & $2.96 \mathrm{E}-28$ & $5.70 \mathrm{E}-26$ \\
\hline 24 & GO_RESPONSE_TO_OXYGEN_CONTAINING_COMPOUND & 1381 & 105 & 0.076 & $5.68 \mathrm{E}-28$ & 1.05E-25 \\
\hline 25 & GO_REGULATION_OF_CELLULAR_COMPONENT_MOVEMENT & 771 & 76 & 0.0986 & $2.37 \mathrm{E}-27$ & $4.20 \mathrm{E}-25$ \\
\hline
\end{tabular}

of phosphorylation led to decrease in G1 phase accompanying an increase in apoptosis or decrease in proliferation in these cells $[10,13]$. This reduction in percentage of cells in the G1 phase alongside a decrease in the proliferation of the cells suggests a cell cycle delay or arrest at $\mathrm{G} 2 / \mathrm{M}$ transition. Our data also demonstrates that WNK1 is strongly phosphorylated upon insulin stimulation in hiPSCs. WNK1 has been identified as a downstream target of phosphorylation by $\mathrm{AKT} / \mathrm{PKB}$ after insulin and IGF-1 stimulation in other cell lines [21, 22]. The cellular significance of this phosphorylation in the context of hiPSC biology would be an interesting avenue for follow-up studies.

To our knowledge, we are the first group to demonstrate the robust regulatory effect of insulin on mRNA transcriptome of hiPSCs. Insulin regulates expression of wide array of genomic targets [23]. Using our starvation protocol, we explored the effect of insulin on gene expression in pluripotent stem cells after $24 \mathrm{~h}$ and $72 \mathrm{~h}$. Our data also indicate that the effect of insulin on the transcriptome at $24 \mathrm{~h}$ and $72 \mathrm{~h}$ seem to be generally in the same direction while becoming more robust overtime.

At the $24 \mathrm{~h}$ time point and as expected, the top 25 identified terms include terms consistent with the role of insulin in cell signaling and transduction. Furthermore, regulation of both cell proliferation and cell death are also among the top 25 identified terms supporting the role of insulin in cell signaling and cellular proliferation of hiPSCs. All these terms remain among the top 100 terms at $72 \mathrm{~h}$.

We compared the differentially expressed genes at 24 $\mathrm{h}$ with identified genes in insulin signaling pathway (KEGG pathway). Among the 9 identified genes, 8 are also differentially expressed at $72 \mathrm{~h}$. Among these 8, there are classic components of insulin signaling 
pathway including INSR, IRS1, IRS2, and CRK. These are expressed at lower levels in the presence of insulin, while signaling suppressor SOCS2 is expressed at higher levels in the presence of insulin. This observation is in line with a negative regulation feedback of components insulin signaling pathway in response to activated insulin signaling or increased sensitivity of hiPSCs cultured without insulin.

We also compared the differentially expressed genes at $24 \mathrm{~h}$ with identified genes involved in regulating pluripotency (KEGG pathway). All of the 7 identified differentially expressed genes are also differentially expressed at $72 \mathrm{~h}$. From these genes, FZD2 and DVL3 belong to Wnt signaling pathway and APC2 is a regulator of the Wnt pathway. Expressions of FZD2 and DVL3 are lower while expression of APC2 is higher in the presence of insulin. These observations suggest that insulin might exert a regulatory effect on Wnt signaling pathway in hiPSCs. The role of Wnt signaling in human pluripotent cells remains controversial [24]. An interesting identified gene is LIF, with a higher expression in the presence of insulin. LIF plays a critical role in maintenance of naïve pluripotent stem cells which represent an earlier developmental stage of pluripotency than hiPSCs (primed stage) [25]. LIF has been shown to increase the proliferation in other human cells $[26,27]$ and is a potential inducer of proliferation in the presence of insulin. Another interesting target is BMP4. Expression of BMP4 appears to be significantly lower in the presence of insulin at both time points. Elevated levels of BMP4 can trigger differentiation of human pluripotent stem cells into mesodermal lineages [28, 29] although differentiation process is suppressed by cell contact [30]. These observations suggest that insulin might maintain hiPSCs at a higher pluripotency stage and less susceptible to differentiation.

In summary, we confirmed that insulin plays a significant role in regulation of proliferation, apoptosis, protein phosphorylation, and mRNA transcription in hiPSCs. Our findings will improve our understanding of the role of insulin in hiPSC biology and are reasonably expected to contribute to better disease modeling experiments affecting the insulin signaling pathways using hiPSCs. We are now focusing on understanding the global transcriptional differences between hiPSCs of insulin-resistant and insulin-sensitive individuals in the presence of insulin.

\section{Additional files}

Additional file 1: Figure S1. RNA sequencing Data QC, related to results section regarding RNA sequencing. (A) Average read quality of each sequencing data. The $\mathrm{x}$-axis shows the average read $\mathrm{Q}$-score and the $y$-axis indicates the number of reads. (B) Base quality along the reads of the sequencing data. The $x$-axis shows the position in the read is plotted and the $y$-axis shows the Q-score is plotted. Median value Q-score are shown by the red lines. The mean value Q-score are represented by dark blue line. The inter-quartile range is shown by the boxplot, while the $10 \%$ and $90 \%$ points are represented by the whiskers. (TIFF $6593 \mathrm{~kb}$ )

Additional file 2: Figure S2. Mapping results, related to the "Results" section regarding RNA sequencing. Samples' summary of mapping results of the reads. In each sample reads could be classified into: mapped (aligned to reference genome), high abundance or out-mapped (e.g., rRNA, mtRNA, polyA, polyC) and unmapped (reads which did not align to anything). (TIFF $6593 \mathrm{~kb}$ )

Additional file 3: Figure S3. Differentially expressed genes in selected $\mathrm{GO}$ pathways related to $24 \mathrm{~h}$ of hiPSC culture with or without insulin, related to Table 1 regarding the top 25 significant GO (biological process) terms. (TIFF 6593 kb)

Additional file 4: Table S1. List of hiPSC lines used. Table S2. Number of identified genes in each sample, related to results section regarding RNA sequencing. Table S3. The 20 most significantly differentially expressed genes at $24 \mathrm{~h}$ of hiPSC culture with or without insulin, related to results section regarding RNA sequencing. The list is ordered by adjusted $p$ values. Average read counts of samples with no insulin treatment are shown in the "baseMean" column. Fold change is the log2 fold change of normalized read count per gene between groups. Followed are the IfCSE (log fold change Standard Error) and stat (Wald statistics). Also listed are $p$ values and adjusted $p$ values corrected for multiple testing using the BenjaminiHochberg False Discovery Rate (FDR) approach. Table S4. The 20 most significantly differentially expressed genes at $72 \mathrm{~h}$ of hiPSC culture with or without insulin, related to results section regarding RNA sequencing. The list is ordered by adjusted $p$ values. Average read counts of samples with no insulin treatment are shown in the "baseMean" column. Fold change is the log2 fold change of normalized read count per gene between groups. Followed are the IfCSE (log fold change Standard Error) and stat (Wald statistics). Also listed are $p$ values and adjusted $p$ values corrected for multiple testing using the Benjamini-Hochberg False Discovery Rate (FDR) approach. Table S5. The top 100 significant GO (biological process) terms associated with differentially expressed transcripts after $72 \mathrm{~h}$ of hiPSC culture with or without insulin, related to results sections regarding RNA sequencing. Table S6. Differentially expressed genes from culture of hiPSCs with or without insulin that have been identified as a part of KEGG signaling pathways regulating pluripotency of stem cells (human). Table S7. Differentially expressed genes from culture of hiPSCs with or without insulin that have been identified as a part of KEGG insulin signaling pathway (human). (DOCX 74 kb)

\section{Abbreviations}

APC2: APC regulator of WNT signaling pathway 2; BMP4: Bone morphogenetic protein 4; DVL3: Disheveled segment polarity protein 3; FGF2: Fibroblast growth factor 2; FZD2: Frizzled class receptor 2; GO: Gene Ontology; hESCs: Human embryonic stem cells; hiPSCs: Human-induced pluripotent stem cells; HPK: Human phosphokinase kinase; IGF1R: Insulin-like growth factor 1 receptor; INSR: Insulin receptor; IRS: Insulin receptor substrate; LIF: Leukemia inhibitory factor; MAPK: Mitogen-activated protein kinase; PI: Propidium iodide; ROCK: Rho-associated kinase; RTK: Receptor tyrosine kinase; SOCS2: Suppressor of cytokine signaling 2;

TGFB1: Transforming growth factor beta 1

\section{Acknowledgements}

We would like to thank Paul Burridge, PhD, Jared Churko, PhD, and Joseph C. Wu, MD, PhD, for the defined medium for culture of hiPSCs, used for our preliminary experiments prior to the reported experiments. Flow cytometry analysis for this project was in part done on instruments in the Stanford Shared FACS Facility.

\section{Authors' contributions}

MS conceived and performed experiments and prepared the manuscript. JK and TQ secured funding and provided expertise, and edited and commented on the manuscript. PC, PL, and AP performed the experiments and contributed to the manuscript revision and discussion. WZ performed data analysis and contributed to the manuscript revision and revision discussions. SLD provided expertise and contributed to the manuscript 
revision and discussion. FA provided expertise. All authors read and approved the final manuscript.

\section{Funding}

This work was supported by NIH grants U01HL107388 and the Diabetes Genomics and Analysis Core of the Stanford Diabetes Research Center (P30DK116074).

\section{Availability of data and materials}

The datasets used and/or analyzed during the current study are available from the corresponding author on reasonable request. RNA-Sequencing data will be available through GEO database.

\section{Ethics approval and consent to participate}

Study is approved by Stanford Institutional Review Board.

\section{Consent for publication}

All subjects gave written informed consent for study participation and publication.

\section{Competing interests}

The authors declare that they have no competing interests.

\section{Author details}

${ }^{1}$ Stanford Cardiovascular Medicine and Cardiovascular Institute, Stanford School of Medicine, Stanford University, Falk CVRC, Room CV273, MC 5406 300 Pasteur Drive, Stanford, CA 94305, USA. ²Department of Developmenta and Regenerative Biology, Icahn School of Medicine at Mount Sinai, Mount Sinai, New York, NY 10029, USA. ${ }^{3}$ Stanford Center for Genomics and Personalized Medicine, Stanford University, Stanford, CA 94305, USA. ${ }^{4}$ Stanford Diabetes Research Center, Stanford University, Stanford, CA 94305 USA. ${ }^{5}$ Genetics Bioinformatics Service Center, Stanford University, Stanford, CA 94305, USA.

Received: 24 October 2018 Revised: 6 May 2019

Accepted: 30 June 2019 Published online: 29 July 2019

\section{References}

1. Takahashi K, Yamanaka S. Induced pluripotent stem cells in medicine and biology. Development. 2013;140(12):2457-61.

2. Iovino S, Burkart AM, Kriauciunas K, Warren L, Hughes KJ, Molla M, et al. Genetic insulin resistance is a potent regulator of gene expression and proliferation in human iPS cells. Diabetes. 2014;63(12):4130-42.

3. Lemonnier T, Blanchard S, Toli D, Roy E, Bigou S, Froissart R, et al. Modeling neuronal defects associated with a lysosomal disorder using patient-derived induced pluripotent stem cells. Hum Mol Genet. 2011;20(18):3653-66.

4. Quarto N, Leonard B, Li S, Marchand M, Anderson E, Behr B, et al. Skeletogenic phenotype of human Marfan embryonic stem cells faithfully phenocopied by patient-specific induced-pluripotent stem cells. Proc Natl Acad Sci U S A. 2012;109(1):215-20.

5. Takahashi K, Tanabe K, Ohnuki M, Narita M, Ichisaka T, Tomoda K, et al. Induction of pluripotent stem cells from adult human fibroblasts by defined factors. Cell. 2007:131(5):861-72.

6. Yu J, Vodyanik MA, Smuga-Otto K, Antosiewicz-Bourget J, Frane JL, Tian S, et al. Induced pluripotent stem cell lines derived from human somatic cells. Science. 2007;318(5858):1917-20.

7. Taniguchi CM, Emanuelli B, Kahn CR. Critical nodes in signalling pathways: insights into insulin action. Nat Rev Mol Cell Biol. 2006;7(2):85-96.

8. Chen G, Gulbranson DR, Hou Z, Bolin JM, Ruotti V, Probasco MD, et al. Chemically defined conditions for human iPSC derivation and culture. Nat Methods. 2011:8(5):424-9.

9. Ludwig TE, Levenstein ME, Jones JM, Berggren WT, Mitchen ER, Frane JL, et al. Derivation of human embryonic stem cells in defined conditions. Nat Biotechnol. 2006:24(2):185-7.

10. Wang L, Schulz TC, Sherrer ES, Dauphin DS, Shin S, Nelson AM, et al. Selfrenewal of human embryonic stem cells requires insulin-like growth factor1 receptor and ERBB2 receptor signaling. Blood. 2007;110(12):4111-9.

11. Carcamo-Orive I, Hoffman GE, Cundiff P, Beckmann ND, D'Souza SL, Knowles JW, et al. Analysis of transcriptional variability in a large human iPSC library reveals genetic and non-genetic determinants of heterogeneity. Cell Stem Cell. 2017;20(4):518-32 e9.
12. Watanabe K, Ueno M, Kamiya D, Nishiyama A, Matsumura M, Wataya T, et al A ROCK inhibitor permits survival of dissociated human embryonic stem cells. Nat Biotechnol. 2007;25(6):681-6.

13. Romorini L, Garate X, Neiman G, Luzzani C, Furmento VA, Guberman AS, et al. AKT/GSK3beta signaling pathway is critically involved in human pluripotent stem cell survival. Sci Rep. 2016:6:35660.

14. Shlyakhtina Y, Pavet V, Gronemeyer H. Dual role of DR5 in death and survival signaling leads to TRAIL resistance in cancer cells. Cell Death Dis. 2017:8(8):e3025.

15. Castrogiovanni C, Waterschoot B, De Backer O, Dumont P. Serine 392 phosphorylation modulates p53 mitochondrial translocation and transcription-independent apoptosis. Cell Death Differ. 2018;25(1):190-203.

16. Holcik M, Yeh C, Korneluk RG, Chow T. Translational upregulation of Xlinked inhibitor of apoptosis (XIAP) increases resistance to radiation induced cell death. Oncogene. 2000:19(36):4174-7.

17. Watanabe S, Umehara H, Murayama K, Okabe M, Kimura T, Nakano T. Activation of Akt signaling is sufficient to maintain pluripotency in mouse and primate embryonic stem cells. Oncogene. 2006;25(19):2697-707.

18. Ho L, Tan SY, Wee S, Wu Y, Tan SJ, Ramakrishna NB, et al. ELABELA is an endogenous growth factor that sustains hESC self-renewal via the PI3KJAKT pathway. Cell Stem Cell. 2015;17(4):435-47.

19. Hossini AM, Quast AS, Plotz M, Grauel K, Exner T, Kuchler J, et al. PI3K/AKT signaling pathway is essential for survival of induced pluripotent stem cells. PLoS One. 2016:11(5):e0154770.

20. Dalton S. Signaling networks in human pluripotent stem cells. Curr Opin Cell Biol. 2013;25(2):241-6.

21. Jiang ZY, Zhou QL, Holik J, Patel S, Leszyk J, Coleman K, et al. Identification of WNK1 as a substrate of Akt/protein kinase $B$ and a negative regulator of insulin-stimulated mitogenesis in 3T3-L1 cells. J Biol Chem. 2005;280(22): 21622-8.

22. Vitari AC, Deak M, Collins BJ, Morrice N, Prescott AR, Phelan A, et al. WNK1, the kinase mutated in an inherited high-blood-pressure syndrome, is a novel PKB (protein kinase B)/Akt substrate. Biochem J. 2004;378(Pt 1:257-68.

23. O'Brien RM, Streeper RS, Ayala JE, Stadelmaier BT, Hornbuckle LA. Insulinregulated gene expression. Biochem Soc Trans. 2001;29(Pt 4:552-8.

24. Sokol SY. Maintaining embryonic stem cell pluripotency with Wnt signaling. Development. 2011:138(20):4341-50.

25. Onishi K, Zandstra PW. LIF signaling in stem cells and development. Development. 2015;142(13):2230-6.

26. Salehnia M, Fayazi M, Ehsani S. Leukemia inhibitory factor increases the proliferation of human endometrial stromal cells and expression of genes related to pluripotency. Int J Reprod Biomed (Yazd). 2017;15(4):209-16.

27. Broholm C, Laye MJ, Brandt C, Vadalasetty R, Pilegaard H, Pedersen BK, et al. LIF is a contraction-induced myokine stimulating human myocyte proliferation. J Appl Physiol (1985). 2011;111(1):251-9.

28. Chadwick K, Wang L, Li L, Menendez P, Murdoch B, Rouleau A, et al. Cytokines and BMP-4 promote hematopoietic differentiation of human embryonic stem cells. Blood. 2003:102(3):906-15.

29. Richter A, Valdimarsdottir L, Hrafnkelsdottir HE, Runarsson JF, Omarsdottir AR, Ward-van Oostwaard D, et al. BMP4 promotes EMT and mesodermal commitment in human embryonic stem cells via SLUG and MSX2. Stem Cells. 2014;32(3):636-48.

30. Tashiro S, Le MNT, Kusama Y, Nakatani E, Suga M, Furue MK, et al. High cell density suppresses BMP4-induced differentiation of human pluripotent stem cells to produce macroscopic spatial patterning in a unidirectional perfusion culture chamber. J Biosci Bioeng. 2018;126(3):379-88

\section{Publisher's Note}

Springer Nature remains neutral with regard to jurisdictional claims in published maps and institutional affiliations. 